\title{
(200) Mis,2a
}

UIILWED STLATEES

DEPARIIIFIT OF THE IIITERIOR

GEOLCGICAI SURVEY

Ground Water Branch

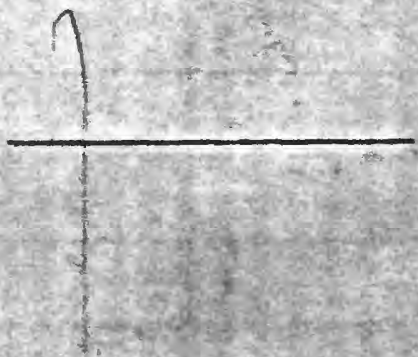

AQUIFER-TEST CONPILATICI FOR THE

MOJAVE DESERI RECICI, CALIFCRIIA
E. ร. Heclelland

Frepared in cooperation with the Californis Department of Water Resources

\author{
OPEW-FIIS DATA REPOET \\ 63.90 \\ Sacramento, Callfornia \\ February 8, 1963 \\ Revised September 30,1963
}

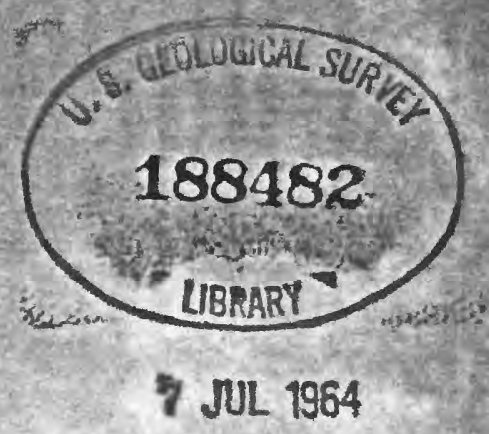




\section{COMTEIISS}

Page

Purpose and scope of this report-

Description of the aquifer-test compliation form-....... 4

Iocation-_.

Pumping data

Well data_-

Summary

Inaian Wells Valley area, aquifer tests_........... II

$\begin{array}{lll}25 S / 39 E-4 R I & 26 S / 39 E-1 I \mathrm{EI}_{I} & 26 \mathrm{~S} / 40 \mathrm{E}-5 \mathrm{PI} I / \\ 25 \mathrm{~S} / 39 \mathrm{E}-12 \mathrm{RI} & 26 \mathrm{~S} / 39 \mathrm{E}-19 \mathrm{I} I & 26 \mathrm{~S} / 40 \mathrm{E}-22 \mathrm{PI} I / \\ 25 \mathrm{~S} / 39 \mathrm{E}-35 \mathrm{NI} I & 26 \mathrm{~S} / 39 \mathrm{E}-24 \mathrm{RI} & 26 \mathrm{~S} / 40 \mathrm{E}-30 \mathrm{E} 2- \\ 25 \mathrm{~S} / 4 \mathrm{OE}-2 \mathrm{OFI} & & \end{array}$

Specific capacity tests in Indian Wells Valley aree........ 16

Antelope Valley area, aquifer tests

$$
11 \mathrm{~N} / 9 \mathrm{~W}-(\text { well } 1 \text { ) } \quad 35 \mathrm{~S} / 36 \mathrm{E}-35 \mathrm{Rl}
$$

Specifle capacity tests in Antelope Valley area_............ 19

Twentynine Palms area, aquifer tests

III/9E-4NI

IN/ $/ 9 \mathrm{E}-5 \mathrm{CI}$

$2 \pi / T E-3 A 1$
$2 N / T E-3 B I$
$3 \pi / T E-35 P C$
$3 N / 8 E-29 I I$
$3 N / 8 E-29 I^{2}$

$2 S / 8 E-6 \mathrm{II}$

Speciflc capacity test3 in Twenty nine Palms area........... 26

Reference IIst_.

1. Tests analyzed but not Included in this compilation because the results were not considered volid.

2. Two tests on one well at different times. 


\section{IILUSTRATIONS}

Page

Figure 1. Index map of Californla showing location of test areas

2. Map showing site of aquifer tests and specific

capacity tests in Indian Wells Valley and

Antelope Valley areas, Californla

3. Map showing site of aquifer tests and specific capacity tests in the Twentymine Palms area, Callfornia 


\title{
AQUIFER-TEST COMPIIATION FOR THE MOJAVE DESERT REGION, CALIFORNIA
}

\author{
By E. J. McClelland
}

\section{PURPOSE AND SCOPE OF THIS REPORT}

This report is the second of a serles the purpose of which is to make available in standard tabular form the results of aquifer tests that have been made by varlous private and public agencies in Calffornia. The scope of the compilation is to describe systematically, in a form agreed upon by the California Department of Water Resources and the Geological Survey, the (1) test location, (2) pumping data, (3) well date and (4) sumary of results. The results of these tests sometimes have been published but most frequently have been used only as a step in obtaining other information, consequently the results and even the location of aquifer tests have not been readily avallable.

This report has been prepered by the Geological Survey under the immediate supervision of Fred Kunkel, district geologist for California, In cooperetion w1th the California Department of Water Resources, and tabulates through November 1962 all tests analyzed by the Geological Survey for the Mojave Desert region (f1gs. I-3). The report is designed to be expanded when additional tests are analyzed or new tests are mede. 


\section{DESCRIPTION OF ACUIFER-TEST COMPILATIOII FOPH}

\section{Location}

We? number.--The test is ident1fted by the number assigned to the pumped well by the California Depertment of Hater Fesources and the feological Survey. The well-numbering system Identifics wells according to the rectangular system for the subdivision of public Iand. That pert of the number preceding the slesh (as in 195/18E-35EI) Indicates the tomship (T. $19 \mathrm{~S}$. ); the number following the slesh is the renge (R. IE E.); the digit following the hyphen is the section (sec. 35); and the letter following the section number Indicates the 40-acre subiivision of the section according to the accompanyling diagram.

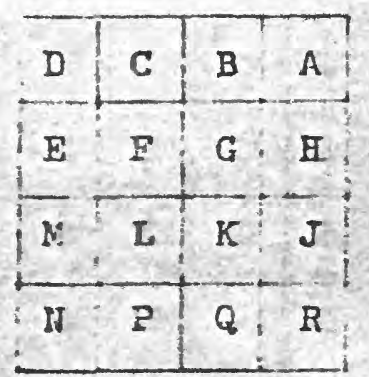

Within each 40-acre tract the wells ere numbered serlaily, as Indicnted by the finel diglt. Following the well number, the Rppropriate base und meridian nre indlcated as follows: H - Humboldt; M - Mt. Dlablo; S - San Bernardino. 
Quadrangle.--Shows the name of the publlshed U.S. Geologtcal Survey topographic map that includes the area of the test, the map scale, and the date $1 t$ was 1saued.

Locstion.--The site of the test 1 s referenced by street names, streems and rivers, cities and county. In some cases, the landowner's name or rame of tenant is included. The ofte of eech test la shown on Plgure 2 and flgure $j$.

Ground-water basin.--The ground-water basin in which the test ras run is ldentifled by name and number of basin and subbasin as assigned by the Callfornia Department of Water Resources.

Ceologic formation.--Geologtc formation, formation members, and named aqufers are 1dentifled.

Date of test.- - Shows the date at start of test followed by duration of pumping in minutes, or hours as indicated.

Agency conducting test.--The agency, and person in charge of fleld test are ldentifled.

Source of teat data.--Shows physical location of the basic data and rame of offices or publlshed report containing data or results of test. In all cases, copies of the basic data are on file with the U.S. Geologlcal Survey, Ground Water Branch, 650 Capltoil Mall, Secramento 14, Callfornia. 
Punpling Data

Purre type, power source and rating.--Shows type or name of pump, source of power, and horsepower rating.

Use.--Use of well at time tested.

Discharge.--Average discharge of pruped vell in gallons per minute during the test.

Maxtmum drawdown.--Total maxtmum drawdown in feet after indlcated pumping time.

Other data.--Any conditions which might affect the accuracy of the proping data test, such a8: changes in purping rates, changes in methods of measuring discharge, influence by other pumpling wells, barometric or recharge effects, or other pertinent 1 tems. 


\section{Well Data}

Well number.--The pumped well is shown on the first I1ne, with observation wells show on subsequent lines.

Depth.--Depth of well in feet.

Perforations.-- Depth of casing perforations in feet, below land surpace.

I.--Distance from prmped well to observation well in feet.

Iog.-- Types of loge, i.e., driller's, electric, etc.

Analysis.--Availability of chemical analysis of well water Indicated by "yes," or "ro."

Water-level measurements.--Ava1lab1l1ty of water-level

measurements during, prior to, or subsequent to the test is indicated as "yes," or "ro."

Other data and remarks.--Physical factors that might affect test results and other information not inclided in the data table. 
Sumery

Purpose of test.-- Purpose as related to determination of aqufer coefficlents, Eround-water movement, storage capacity, underflow conditions, foundation design, and related items.

Aquifer thickness.--Thickness in feet.

Agulfer saturated thickness.--Th1ckness in feet.

Specific capac1ty.--Rate of yleld of the pumped well in gallons per mute per foot of drawdown.

U.S. Geological Survey formation yfeld factor.--

Formation yleld factor $=\frac{\text { Specific capacity } \times 100}{\text { Aquifer saturated thickness }}$

Method of analysis.--Irdicates solution utilized such as: equilibrium, ronequilibrium, leaky aquifer, etc.

Coefficlent of transmissiblilty.--Expressed as the rate of flow of water, in gallons per day, at the prevalling water temperature through each vertical strip of the aquifer on foot wide, having a hefght equal to the saturated thickness of the aquifer and under a unit hydraulic gradient.

Coefficient of permeability.--Expressed as the rate of flow of water in gallons per day through a vertical cross section of one square foot under a unit hydraulic gradient. 
Coefficlent of storage.-- The volume of water an aquifer releases from or takes into storage per unit of surface area of the aquifer per unft change in the component of head normal to that surface is called the coefficient of storage.

Test evaluat1on.--Irdicates overall validity of teat, 1.e., excellent, good falr, or poor, along with sumery of reasons. In general, the criteria used by the author for judging 1s: poor tests are all test for which the pumping cycle was 100 minutes or less, or tests in which the response of observation wells was small or erratic. A poor teat does rot necessarily imply poor fleld technique. Falr tests are test. for which the proping cycle was 100 to 500 minutes and test results are judged better than poor. All tests involving a pumped well only are graded poor or fair unless the pumped-well data are exceptionally detalled, and involve both the drawdown and recovery cycle. Good tests are tests for which the pumping cycle was 500 minutes or more and proped-trell data as referred to above. Excellent tests are tests for which the pumpling cycle was 500 minutes or more, with two or more properly spaced observation wells, detailed and accurate observation well and punped-well data were obtained and results of wells support each other. 
Some tests show in the "Contents" are not lincluded in the compllation because the results were considered to be unsatisfactory. They are listed to Indicate that data exist and are avallable in the flles for examination. 


\section{U.S. GEOLGICAL SURVEY \\ TRCUTO HATER 3RANCH \\ AGUTME-TKET CCMPIIATICR}

elI $25 \mathrm{~S} / 39 \mathrm{E}-4 \mathrm{RI} \quad \mathrm{M}$ Ruedrangle Ifttle Iake, $15 \mathrm{min.} 1954$

icction About 4 miles east of Brown, Kern County

Fround water besin Indian Wells Valley 6-54.00

sealogic Formation Allurifum

Jate or rest Dct. 22, $195367 \mathrm{hr}$, ixency Enduct:ng Test USG8, Fred Kunkel

Source or Test Data USCS, Sacramento

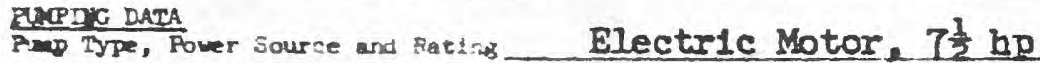

Uee Public Supplyoischarge $145 \mathrm{gm} \quad 1.97$ ft. arter 2870 min.

other data

\begin{tabular}{|c|c|c|c|c|c|c|c|c|}
\hline \multirow{2}{*}{ Hell No. } & \multirow{2}{*}{ Depth } & \multirow{2}{*}{ Ferforat: a $x$} & \multirow{2}{*}{$r^{*}$} & \multirow{2}{*}{$\omega$} & \multirow{2}{*}{ Anaiysis } & \multicolumn{3}{|c|}{ water-level measurements } \\
\hline & & & & & & Dradoum & Recovery & Histortcel \\
\hline $25 / 39-4 R I$ & 200 & $\infty$ & $-\infty$ & Dr1llers & уев & yes & yes & res \\
\hline & & & & & & & - & \\
\hline$=$ & & & & & & & & \\
\hline - & & & & & & & & \\
\hline
\end{tabular}

Ceber Data and Remarke

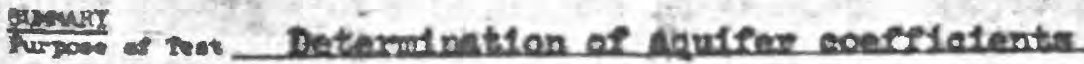

aquifer mickes: $143 \mathrm{ft}$

-eci:1- apacity 13
Aquíer intureted mickress

Fis Pormatzon Yiels Pactor
143 ft

51

4.met of ara jass _ _. Nonequ111br1um

proverue of 87,000

terma sit $610 \mathrm{gpd} / \mathrm{st}$

cerencient of storsen

Elr, arsinom data not usable 
U.S. iECLLGICAL SURYEY

GFCUND NATER AFAMTH

AQUTREF- TXT CCMPIEATIS

25S/39E-12RI H Hountain Springs Canyon, 15 min., 1953

wation About 10 miles northeast of Inyokern, Kern County

Srownit weter Basin Indian Wells Valley, 6-54.00

seologic Farsetion Alluvium

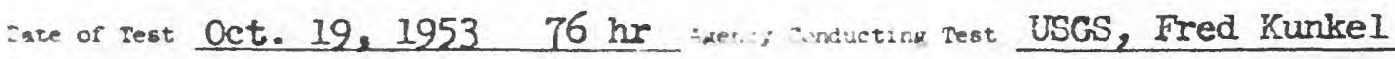

source or Teat Dats USCS, Sacramento

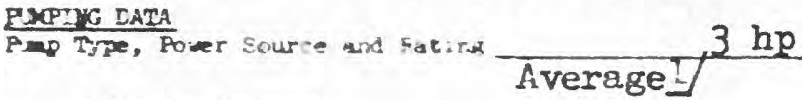

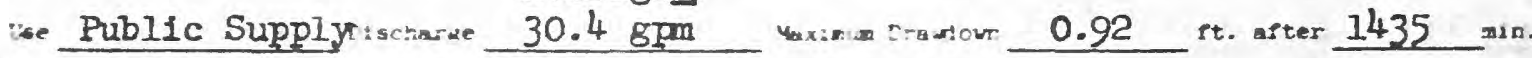

ther tare 1. Discharge averaged $34.0 \mathrm{gpm}$ for first $1470 \mathrm{~min}$. then reduced to an average of $28.3 \mathrm{gpm}$ for an additional $2480 \mathrm{~min}$. Pumping stopped after $3950 \mathrm{~min}$.

-E:- EATA

\begin{tabular}{|c|c|c|c|c|c|c|c|c|}
\hline \multirow{2}{*}{ e.: io } & \multirow{2}{*}{ epers } & \multirow{2}{*}{ serfura: :one } & \multirow{2}{*}{$r^{*}$} & \multirow{2}{*}{ 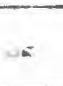 } & \multirow{2}{*}{$\sin -y 5$ is } & \multicolumn{3}{|c|}{-acer-evel neasuremerits } \\
\hline & & & & & & Eradour & Secovery & H:atortca: \\
\hline $25 / 39-12 R I$ & 180.5 & -- & -- & -- & yes & yes & yes & yes \\
\hline & & & & & & & & \\
\hline & & & & & & & & \\
\hline & & & & & & & & \\
\hline & & & & & & & & \\
\hline
\end{tabular}

ino Dete ard Gemarks.

Rurpose of rot Detemination of aguffer coefficients

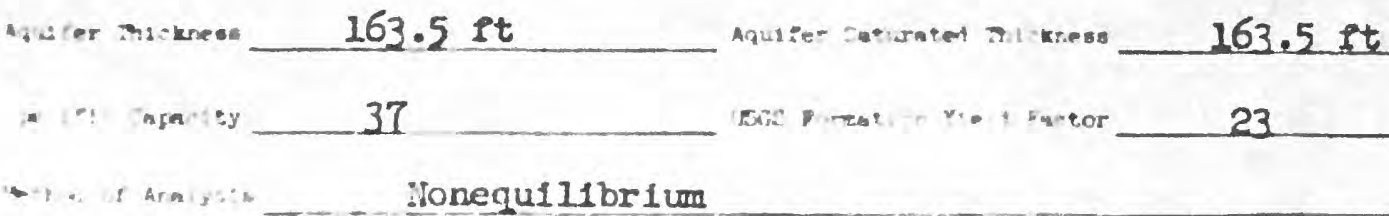

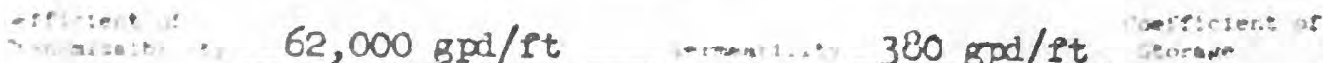
Poor, dr:iom dato are not reliable. 


\title{
U.S. TECLCG:CAI SURYEX \\ GRCLATO WATEF 3RANCH \\ AJUTFR-TSTT CCMPIIATICN
}

\begin{abstract}
LOCATIC 25S/39E-35NI M sumdrangie Inyokern, I5 min., 1943
ocation About $5 \frac{1}{2}$ miles northeast of Inyokern, Kern County
\end{abstract}

Ground water Basin $\frac{\text { Indian Wells Valley, 6-54.00 }}{\text { Alluvium }}$

Late of Test July 29, $1953 \quad 205 \mathrm{hr}$ 2.ersy conduct:ins Test USGS, Fred Kunkel

Source or Test tate USGS (GW), Sacramente

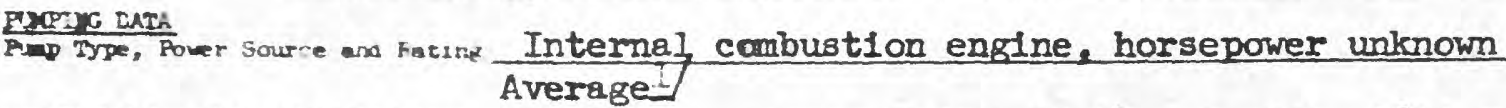

use Public Supply Disctarke 275 gPm Taxim aradown 13.24 it. arter $2810 \mathrm{~min}$.

other a 1. Discharge varied from about 225 gpm to about $300 \mathrm{gpm}$, in general increasing with proping time HELI IAIA

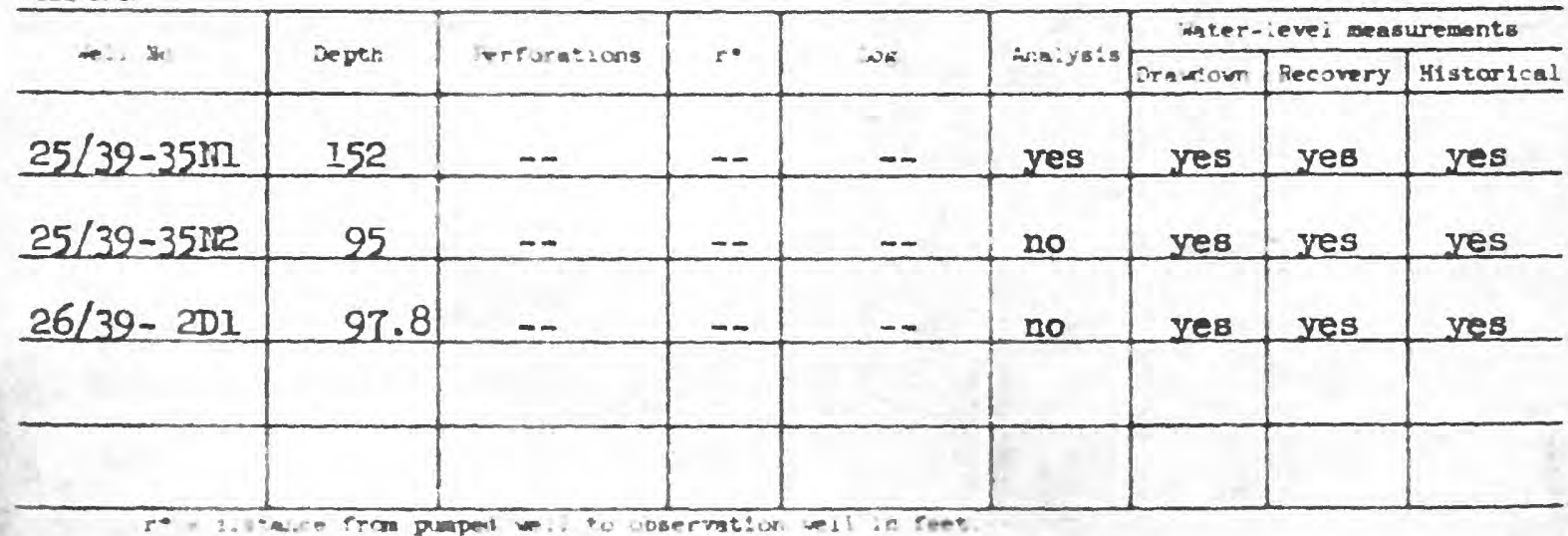

cener Dats are Nemartus

\section{rumar}

hurpace of zere

Determination of soutfer coerreclents

Aquster micknees

25 tt

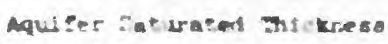

95 it

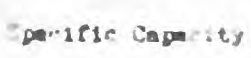

21

E. pormetion 11. 1 Pator

22

-iring of Ariniosite

Nonequilibrlum

- meriere or Averago

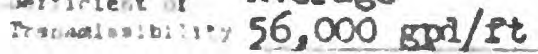

$\rightarrow=270 \mathrm{god} / \mathrm{ft}$

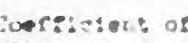

0.01 -

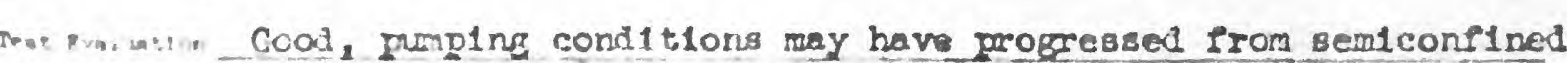
to irater table durlas the test. 
DOCATIN 265/39E-IIE1 M Inyokern, $15 \mathrm{~min} ., 1943$

Lacion About $3 \frac{1}{2}$ miles northeast of Inyokern, Kern County

Ground water Basin Indian Viells Valley, 6-54.00

Geologie Fornation Alluvium

Date of Test July 29, $1953 \quad 114 \mathrm{hr}$ ake:scy Conduc:sing Test USCS, Fred Kunkel

Sourse of Test Data USGS (GW), Sacramento

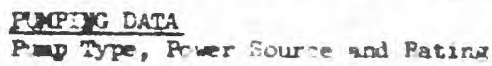

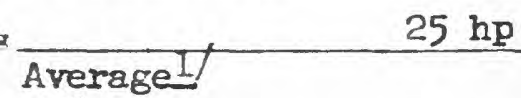

Use Public Supply D1scharge $218 \mathrm{gpm}$ Maximum Iradicum 6.65 ft. after 3590 min.

Tther at 1. Pump speed slow during first 24 hrs of test, discharge $2918 \mathrm{gm}$ at end of test.

WELi DATA

\begin{tabular}{|c|c|c|c|c|c|c|c|c|}
\hline \multirow{2}{*}{ det. } & \multirow{2}{*}{ repth } & \multirow{2}{*}{ Ferforations } & \multirow{2}{*}{$r *$} & \multirow{2}{*}{ ine } & \multirow{2}{*}{ Mra:ysis } & \multicolumn{3}{|c|}{ water-ievel measurements } \\
\hline & & & & & & Cradour. & Recovery & Historical \\
\hline $26 / 39-11 E I$ & 250 & -- & -- & Drillers & Yes & IJO & Yes & Yes \\
\hline & & & & & & & & \\
\hline & & & & & & & & \\
\hline & & & & & & & & \\
\hline & & & & & & & & \\
\hline
\end{tabular}

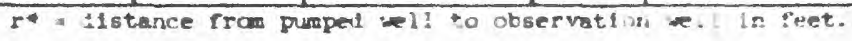

Cther Date and Gerarks

Sumupy

Aquifer Thickuess $\frac{148 \mathrm{ft}}{33}$ Aquiter cuturated mischean $\frac{148 \mathrm{ft}}{22}$

$\therefore$........ Poor, no irawiown datal rivallab!e. 


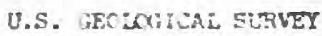

GECUND WATES BKANCH

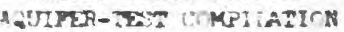

Weli no. 26S/39E-24RI M Peadrargie Ridgecrest, 15 min., 1953

Location 4 miles west of Chins Lake, Kern County

Ground weter indian Wells Valley, 6-54.00

Seologic Pormation AlIuvilum

Date of Iest Har. 2, $195398 \mathrm{hr}$ Agency Conducting Test USCS, Fred Kunkel

Source of test Data USCS (GW), Sacramento

RMPTYG DATA

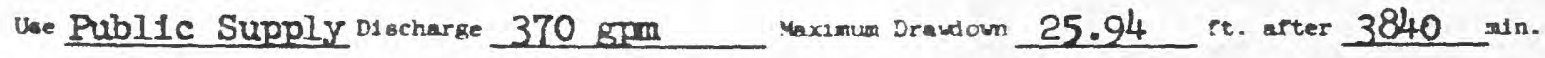

Other data

\begin{tabular}{|c|c|c|c|c|c|c|c|c|}
\hline \multirow{3}{*}{ Te.t so. } & \multirow{3}{*}{ repth } & \multirow{3}{*}{ Perforatione } & \multirow{3}{*}{$r^{*}$} & \multirow{3}{*}{.00} & \multirow{3}{*}{ Ana:ysís } & \multirow{2}{*}{\multicolumn{3}{|c|}{ Whter-ievel neasurements }} \\
\hline & & & & & & & & \\
\hline & & & & & & Draviom & Recovery & Histarice. \\
\hline $26 / 39-24 R]$ & 480 & $\begin{array}{l}160-281 \\
412-460\end{array}$ & -- & Drillers & yes & yes & yes & yes \\
\hline $26 / 39-24 \pi I$ & 323 & $\begin{array}{l}190-197 \\
230-301\end{array}$ & 1515 & Drillers & yes & yes & yes & yes \\
\hline $26 / 39-2421$ & 361 & $\begin{array}{r}18-285 \\
325-345 \\
\end{array}$ & IOT2 & Drillers & yes & yes & yes & yes \\
\hline 26/40-19M & 306 & & 1180 & Drillers & yes & yes & yes & yes \\
\hline 26/40-19PI & 261 & $\begin{array}{l}192-220 \\
253-259\end{array}$ & 1998 & Drillers & yes & yes & yes & yes \\
\hline
\end{tabular}

Otber Data and Remarks 26/39-25D2 ( $r=4250 \mathrm{ft}$ ) also observed but too far from

pumped well.

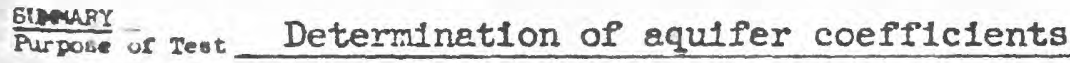

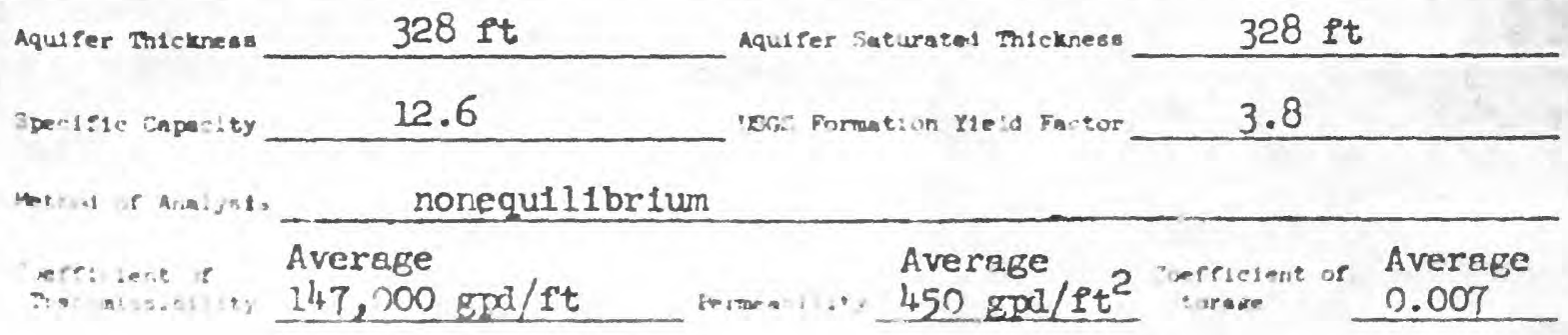

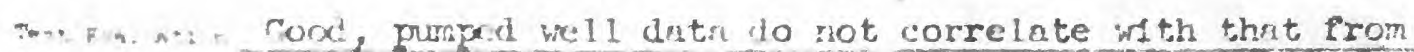

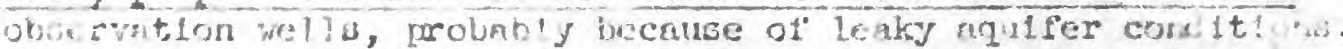


Speciffc Cspacity Tests in Irdian Wells Valley Area

\begin{tabular}{|c|c|c|c|c|c|}
\hline Weil number & $\begin{array}{l}: \\
: \text { WelI cepth } \\
: \quad(\text { Seet }) \\
:\end{array}$ & $\begin{array}{l}\text { : Date test } \\
: \\
:\end{array}$ & $\begin{array}{l}\text { : } \\
: \text { ischarge } \\
: \quad(\text { gpm }) \\
:\end{array}$ & $\begin{array}{l}: \\
: \text { Drandown } \\
:(\text { feet }) \\
:\end{array}$ & $\begin{array}{l}: \text { Speciffc } \\
: \quad \text { capacity } \\
:(\mathrm{gpm} / \mathrm{ft} \text { of } \mathrm{dd})\end{array}$ \\
\hline 24/40-200I & 28.3 & $5-22-53$ & 5 & 9.5 & 0.5 \\
\hline $25 / 39-4 \mathrm{RI}$ & 200 & $: 0-21-53$ & 145 & 2.0 & 73 \\
\hline$I 2 R I$ & 180.5 & $=0-20-53$ & 34 & 0.9 & 37 \\
\hline $35 \mathrm{NI}$ & 152.0 & $7-29-53$ & 275 & 13.2 & 21 \\
\hline $25 / 40-20 F 1$ & 132.6 & $10-21-52$ & 96 & 4.4 & 22 \\
\hline $26 / 39-1 E I$ & 250 & 1920 & 450 & 20 & 22 \\
\hline $5 F 1$ & 200 & $\begin{array}{l}8-7-52 \\
8-7-52 \\
8-8-52 \\
8-9-52\end{array}$ & $\begin{array}{l}450 \\
650 \\
910 \\
830\end{array}$ & $\begin{array}{r}9 \\
13 \\
20 \\
18\end{array}$ & $\begin{array}{l}50 \\
50 \\
46 \\
46\end{array}$ \\
\hline$I Z E I$ & 250 & $7-29-53$ & 278 & 6.65 & 33 \\
\hline $19 K I$ & 803 & $10-6-60$ & 3,500 & 74 & 47 \\
\hline 19P1 & 446 & $\begin{array}{l}8-16-44 \\
9-7-45\end{array}$ & $\begin{array}{l}2,500 \\
1,980\end{array}$ & $\begin{array}{l}7 \\
6.7\end{array}$ & $\begin{array}{l}358(?) \\
296(?)\end{array}$ \\
\hline 1901 & 367.5 & $\begin{array}{l}3-14-44 \\
9-7-45 \\
3-19-53 \\
3-20-53\end{array}$ & $\begin{array}{r}750 \\
295 \\
2,670 \\
785\end{array}$ & $\begin{array}{c}13 \\
2.7 \\
18.96 \\
7.91\end{array}$ & $\begin{array}{l}58 \\
105(?) \\
84 \\
99\end{array}$ \\
\hline $20 F^{1} 1$ & 333 & $10-17-58$ & 818 & 18 & 45 \\
\hline $23 \mathrm{JI}$ & 800 & $10-17-60$ & 3,560 & 79 & 45 \\
\hline $24 K$ & 323.1 & $\begin{array}{l}6-20-44 \\
9-7-45\end{array}$ & $\begin{array}{r}1,000 \\
350\end{array}$ & ${ }^{7} .4$ & $\begin{array}{c}143(?) \\
28\end{array}$ \\
\hline $24 i n$ & - & $10-26-60$ & 3,800 & 63 & 63 \\
\hline $24 P 1$ & 825.0 & $3-6-58$ & 2,750 & 33.4 & 82 \\
\hline $24 a 1$ & 361 & $11-8-44$ & 800 & 30 & 28 \\
\hline
\end{tabular}


Specific Capacity Tests in Indian Wells Valley Area--Continued

\begin{tabular}{|c|c|c|c|c|c|}
\hline $\begin{array}{r}\text { Vel1 number: } \\
:\end{array}$ & $\begin{array}{l}: \\
: \text { Well depth } \\
: \quad \text { (feet) } \\
:\end{array}$ & $\begin{array}{l}\text { :Date teste } \\
: \\
\end{array}$ & $\begin{array}{l}\text { : } \\
: \text { ischarge } \\
: \quad \text { (gpm) } \\
\end{array}$ & $\begin{array}{l}: \text { : } \\
:(\text { feet }): \\
: \quad:\end{array}$ & $\begin{array}{c}\text { Specific } \\
\text { capacity } \\
(\mathrm{g} \mathrm{pm} / \mathrm{ft} \text { of } \mathrm{dd})\end{array}$ \\
\hline $26 / 39-24 R I$ & 480 & $\begin{array}{l}4-20-44 \\
9-7-45 \\
3-5-53\end{array}$ & $\begin{array}{l}900 \\
328 \\
370\end{array}$ & $\begin{array}{l}22 \\
11.5 \\
25.94\end{array}$ & $\begin{array}{l}41 \\
28 \\
12.6\end{array}$ \\
\hline $25 \mathrm{D2}$ & 330 & $\begin{array}{ll}5- & -50 \\
9- & -50\end{array}$ & $\begin{array}{l}180 \\
355\end{array}$ & $\begin{array}{c}4 \\
10.5\end{array}$ & $\begin{array}{l}45 \\
34\end{array}$ \\
\hline $25 E 1$ & 387 & $\begin{array}{ll}5- & -51 \\
7- & -51 \\
3- & -52\end{array}$ & $\begin{array}{r}230 \\
320 \\
150\end{array}$ & $\begin{array}{l}5 \\
7 \\
4\end{array}$ & $\begin{array}{l}46 \\
46 \\
38\end{array}$ \\
\hline 2202 & 364 & $2-11-58$ & 419 & 6.3 & 66 \\
\hline $30 \mathrm{Cl}$ & -- & $9-7-45$ & 126 & 2.3 & 55 \\
\hline $30 F I$ & 619 & $9-7-45$ & 2,200 & 27 & 81 \\
\hline $26 / 40-1 A 2$ & - & $3-17-54$ & 35 & 27 & 1.3 \\
\hline 19111 & 306 & $9-7-45$ & 450 & 31.7 & 14 \\
\hline $19 P 1$ & 261.0 & $11-8-44$ & 700 & 39 & 18 \\
\hline 20 ir & 190.1 & $9-7-45$ & 127 & 13 & 10 \\
\hline $28 J 1$ & -- & $12-30-48$ & 152 & 32 & 4.8 \\
\hline $30 E 2$ & 402 & 1954 & 1,680 & 50 & 34 \\
\hline $33 \mathrm{Al}$ & 400 & $\begin{array}{l}1950 \\
1950\end{array}$ & $\begin{array}{l}850 \\
250 \\
269\end{array}$ & $\begin{array}{r}53 \\
8 \\
8\end{array}$ & $\begin{array}{l}16 \\
31 \\
34\end{array}$ \\
\hline $33 P 2$ & 130 & $8-6-59$ & 385 & 21 & 18 \\
\hline 34111 & 232 & $9-7-44$ & 1,020 & 21.9 & 47 \\
\hline $36 \mathrm{AI}$ & 270.0 & $2-8-54$ & 75 & 6.3 & 12 \\
\hline $27 / 40-4 \pi 7$ & 252 & $\begin{array}{ll}7- & -50 \\
4-2-53\end{array}$ & $\begin{array}{r}1,400 \\
950\end{array}$ & $\begin{array}{l}30 \\
22\end{array}$ & $\begin{array}{l}47 \\
43\end{array}$ \\
\hline $2 \varepsilon / 3 \tilde{C}-1 E F$ & 930 & $3-2-53$ & 50 & 250 & 0.3 \\
\hline
\end{tabular}




$$
\begin{aligned}
& \text { U.S. IEOLCGICA: SLRVEY } \\
& \text { GREUN WATER MRAACH } \\
& \text { AQUIFTF-TEST CCMPILATICN }
\end{aligned}
$$

weit no. IIN/9w-(weII I) S rumirarge Castle Butte SE, $7.5 \mathrm{~min}, 1947$

weation About $6 \frac{1}{2}$ miles west of Boron, Kern County, exact location not identifled by owner.

Ground Weter Basin Antelope Valley, Iorth Muroc Bes1n, 6-44.08

Geologie Pormetion Alluvium

Date of Test Aus. 14, $1955262 \mathrm{hr}$ Agency Conductire Test Owmer

Source of Test Data USGS (GW) Sacremento

PROPTIS DATA

Pag Aype, Fower Source and Fating

Average

\begin{tabular}{|c|c|c|c|c|c|c|c|c|}
\hline \multirow{2}{*}{ Heil No. } & \multirow{2}{*}{ Septh } & \multirow{2}{*}{ Pertoracions } & \multirow{2}{*}{$r^{\bullet}$} & \multirow[b]{2}{*}{ Loe } & \multirow{2}{*}{ Ara:ysis } & \multicolumn{3}{|c|}{ nter-ievel measurements } \\
\hline & & & & & & Draved oum & Pecovery & Historical \\
\hline WeII 1 & -407 & $96-407$ & -- & Drillers & yes & yes & yes & yes \\
\hline WelI 2 & 372 & $110-336^{2}$ & 504 & - & no & yes & yes & yes \\
\hline Well 3 & 382 & -- & 1012 & -- & no & yes & yes & no \\
\hline 31 & & & & & & & & \\
\hline & & & & & & & & \\
\hline
\end{tabular}

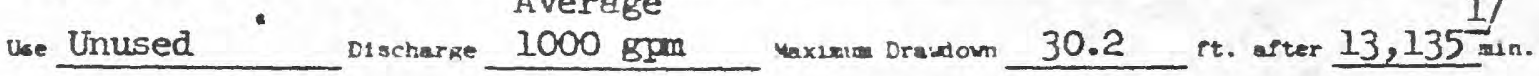

other dat 1. Total pumpling time $219 \mathrm{hr}$, recovery measured for $43 \mathrm{hr}$.

cther Data na: 4mark 2. Cased to $348 \mathrm{ft}$. 3. Well $4(x=2463)$, well 5 $(r=2230)$, well $6(r=3404)$ measured but not analyzed becuase of small whter-level changes and bnrometric fluctuations.

sumeng or ses Deternination of aquifer coeferetents

nquier miekrese 311 Aquiser inturated aciclues: 300 33.1

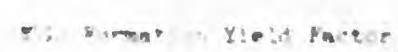
10

Nonequil1brium

Approx.

$20,000 \mathrm{sgd} / \mathrm{ft}$

Gool, leak aquifer and lyb1s indleutes $m=200,000 \mathrm{gpl} / \mathrm{ft}$.

Because of poosible leskate, coefflclent of atorage ondt. 


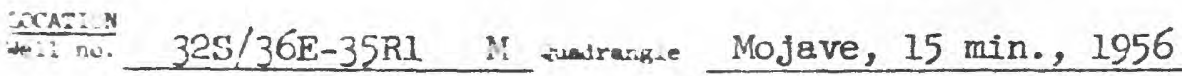

wention About 7 miles northeast of Mojave, Kern County

Ground water Basin Antelope Valley, Chaffee Basin, 6-44.04

Geologic Formation Alluvium

Date or Test Dec. 26, $1956114 \mathrm{hr}$ igetiry coniuctirag rest USGS, L. C. Dutcher

Source of Test Data USGS (GW), Sacramento

RAPIC DATA

Pap TYPe, Power Source and Fatir.d

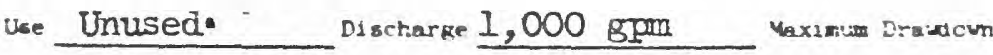
Pt. after min.

Otber data

\begin{tabular}{|c|c|c|c|c|c|c|c|c|}
\hline \multicolumn{9}{|l|}{ - ELL DATA } \\
\hline \multirow{2}{*}{ स⿺ } & \multirow{2}{*}{ Septs } & \multirow{2}{*}{ Perforations } & \multirow{2}{*}{$r$} & \multirow{2}{*}{ in } & \multirow{2}{*}{ Ascalys is } & \multicolumn{3}{|c|}{ dater-ievel measurements } \\
\hline & & & & & & Crajodour: & Recovery & Histor $1 \mathrm{Caj}$ \\
\hline $32 / 36-35 R I$ & 800 & $333-694$ & - & Drillers & Yes & ITO & No & \\
\hline $32 / 36-35 R 2$ & 720 & $220-720$ & 300 & Drillers & No & Yes & Yes & \\
\hline & & & & & & & & \\
\hline & & & & & & & & \\
\hline & & & & & & & & \\
\hline
\end{tabular}

ther Dats and Fersiks Depth to water before pumping 211.76 ft below measuring point in $35 R^{2}$

Sumargr

Aquilfer Thirkeress Aquifer aturntens mickrexs

Le: :1- inpe:ty
hering 5 Arming: nonequilivr1un

$\because \ldots \quad 1,500,000 \mathrm{gpl} / \mathrm{ft}$

Imor, test min not be mil1d, the observed well probably is not

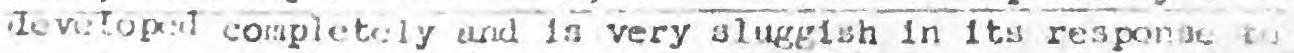
puratour 78 
Epecific Capacity Tests in Antelope Valley Are日

\begin{tabular}{|c|c|c|c|c|c|}
\hline $\begin{array}{r} \\
\text { Well number: } \\
\vdots \\
\end{array}$ & $\begin{array}{l}\text { Well depth: Date t } \\
(\text { feet }): \\
\vdots\end{array}$ & $\begin{array}{c}: \\
\text { testec } \\
\vdots \\
: D \\
\end{array}$ & $\begin{array}{c}\text { Discharge: } \\
(\text { gprs }): \\
\vdots\end{array}$ & $\begin{array}{l}: \\
: \text { Drawdiown: } \\
:(\text { feet }): \\
\vdots \quad:(8\end{array}$ & $\begin{array}{c}\text { Specific } \\
\text { capacity } \\
\text { (gpm/ft of dd) }\end{array}$ \\
\hline $9 / 8-6 \mathrm{mn}$ & 467 & & 1,340 & 10 & 134 \\
\hline $6 \mathrm{E} 2$ & 354 & & 1,000 & 6 & 166 \\
\hline $9 / 9-6 A I$ & 199 & & 330 & 12 & 28 \\
\hline $62 ?$ & 112 & & 254 & 59 & 4.3 \\
\hline ELI & 147 & & 149 & 10 & 25 \\
\hline - $6 n$ & 126 & & 75 & 23 & $3 \cdot 3$ \\
\hline $18 \mathrm{Cl}$ & 360 & & 230 & 24 & 10 \\
\hline 24III & 315 & & 300 & 95 & 3.2 \\
\hline $9 / 10-24 \mathrm{CI}$ & 750 & & 1,350 & 101.6 & 13 \\
\hline 10/9- 7AI & 200 & & 50 & 9 & 5.6 \\
\hline TA2 & 200 & & 100 & 8 & 12 \\
\hline $10 / 12-12: 1$ & 249 & & 270 & $60(?)$ & 4 \\
\hline $20 C I$ & 161 & & 73 & 27 & 2.7 \\
\hline $26 F 1$ & 140 & & 225 & 80 & 2.8 \\
\hline $10 / 13-14 Q 1$ & 463 & & 360 & 90 & 4.0 \\
\hline $11 / 9-36 \mathrm{Cl}$ & 407 & & 1,000 & 30.2 & 33 \\
\hline $11 / 10-36 \mathrm{mIn}$ & 320 & & 206 & 68 & 3.0 \\
\hline $11 / 12-26 \mathrm{~J} 2$ & 361 & & 1,200 & 60 & 20 \\
\hline $32 / 36-2101$ & 805 & & 263 & 2.3 & 114 \\
\hline
\end{tabular}

* Lates tested unknown. 
U.S. IENLUECAL SLAVEY

TACLAC $\triangle A$ IER BFANCH

AQUTFE-IEST COMPILATICR

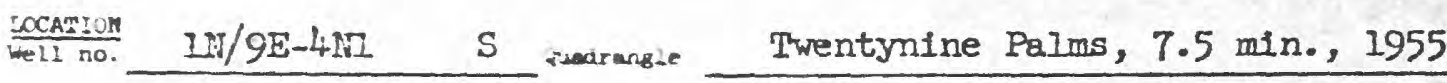

Location About 4 miles north of Twentymine Palms, San Bernardino County

Ground water Basin Colorado River Basin, 7-

Ceologic Formation Alluvium

Date of teat Apr. 7, 1952 If ABency Conductirg Teat Arehitects \& Engineers

Source of Teat Date USGS (GW), Sacramento

W. O. Wagner

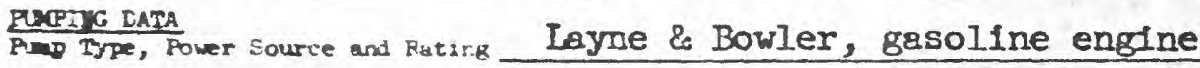

use Public Supplyoischarge If Maximum Drawiom 41.00 it. after 1982 min.

other det 1. Pumped for about $33 \mathrm{hr}$ at discharge rates starting at $51 \mathrm{gpm}$ and increased in five steps to $256 \mathrm{gpm}$.

WELI DATA

\begin{tabular}{|c|c|c|c|c|c|c|c|c|}
\hline \multirow{2}{*}{ nel. Yo. } & \multirow{2}{*}{ Septr: } & \multirow{2}{*}{ Ferforations } & \multirow{2}{*}{$r$} & \multirow{2}{*}{ weng } & \multirow{2}{*}{ Ans:ysis } & \multicolumn{3}{|c|}{ hater-level measurements } \\
\hline & & & & & & Draidoum & Recovery & Histortcel \\
\hline $2 \mathrm{~N} / 9 \mathrm{E}-4 \mathrm{NI}$ & 136 & - & - & Drillers ${ }^{2}$ & no & yes & yes & yes \\
\hline & & & & & & & & \\
\hline & & & & & & & & \\
\hline & & & & & & & & \\
\hline & & & & & & & & \\
\hline
\end{tabular}

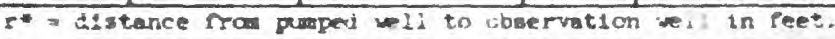

other Data and Bemark 2. Avallable from Mogle Bros., drillers, Chino, Calif.

Prepumping water level in $4 \mathrm{mI}, 12.0$ ft below land surface.

Sumprir or Test Test measurements were a secondary product of pumping to develop the well.

Aquuser Thicknes: Aqut:er satiurated m: moness

Eperiri- capeity $6.2^{3 / 1}$ TCS Pormation Ytela Faceor

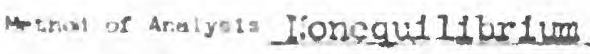

- ipeliene or Approx.

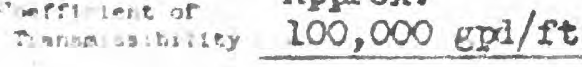
inmentilty

The fvalue Io evaluation because of varted diccharge 
LCAT:CN $1 \mathrm{~W} / 9 \mathrm{E}-5 \mathrm{GI} \quad \mathrm{S}$ Tuarrangle Twentymine Palms, $7.5 \mathrm{~min} ., 1955$

iocation About 4 miles north of Twentynine Palms, San Bernardino County

Ground hater Basin Colorado RIver Basin, 7-

Geologic Farmation Alluvium

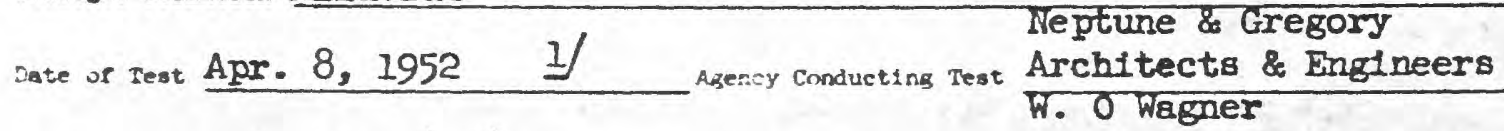

Source of Test Dats USGS (GW), Sacramento

PUP ISpe, Power Source and Fating Layme \& Bowler, electric motor, 25 hp

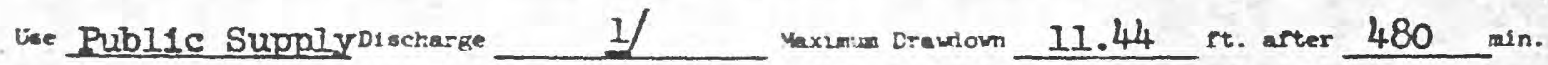

other date I. Pumped for $482 \mathrm{~min}$. at discharge rates starting at $80 \mathrm{gmm}$

and Increased in four steps to about $275 \mathrm{gmm}$.

-ELL DATA

\begin{tabular}{|c|c|c|c|c|c|c|c|c|}
\hline \multirow{2}{*}{ deis ko. } & \multirow{2}{*}{ Depth } & \multirow{2}{*}{ Perforations } & \multirow{2}{*}{$r^{\circ}$} & \multirow{2}{*}{$\cos \theta$} & \multirow{2}{*}{ Anelysis } & \multicolumn{3}{|c|}{ Water-leve! measurements } \\
\hline & & & & & & Dravdom & Recovery & H1storical \\
\hline $1 \pi / 9 \mathrm{E}-5 \mathrm{CI}$ & 500 & $=-$ & - & Dr111ers 2 & no & yes & yes & yes \\
\hline & & & & & & & & \\
\hline & & & & & & . & & tet \\
\hline & & & & 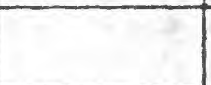 & & & & \\
\hline
\end{tabular}

other tata and Rrarks 2. Avallable from Mogle Bros., drillers, Chino, Callf.

Prepumplng water level in $5 \mathrm{Gl}$. 5.11 et below land surface.

Bumpary of Test Iest measurements were a secondary product of pumpling to develop well.

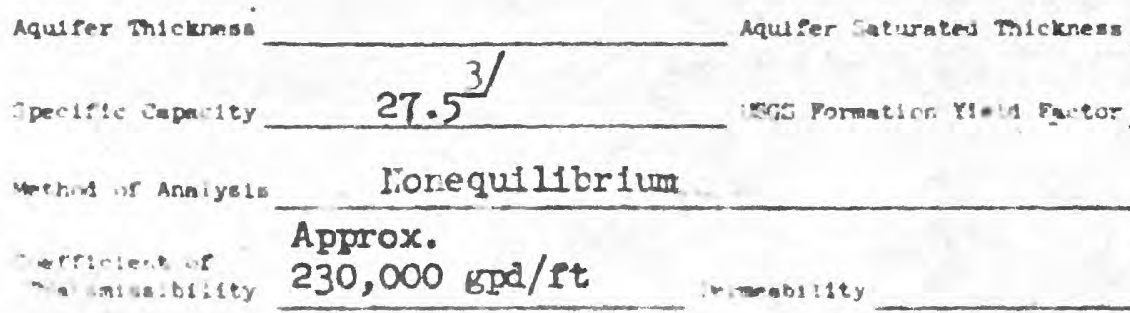

em notur - Ho evaluation becauce of mrled discharge.

3. At maxlinem d1scharge 


\section{U.S. GECLUICAL SERVY \\ WRCUNC WATER BRANCH

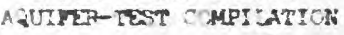

LOCATYCI $2 \mathrm{TH} / \mathrm{TE}-3 \mathrm{AI}$ Puadrangle Deadman Lake SW, 7.5 min., 1955

Locetion About 15 miles northwest of Twentynine Palms, San Bernardino County

Ground Water Basin Colorado River Basin, 7-

Ceologic Forsation AlIuvLum

Date of Test Jan. 31, 1953 IJ Agerscy Conductirig Test USGS, F. S. R1ley

Source of Test Data USCS (GW) Sacremento

PXPTIG ENTA

Pind Type, fover Source and Fisting

Use Public Supplydischarge

1 Maxioum Drasciown 50.72 th. apter $1064 \mathrm{~min}$.

other dot 1. Pumped for about 27 hr at discharge rates varying from about $900 \mathrm{gpm}$ to about $1900 \mathrm{gpm}$. Rechecked July $23(985 \mathrm{gpm})$ and Aug. 6, 1953 (870 g gm).

WELL SATA

\begin{tabular}{|c|c|c|c|c|c|c|c|c|}
\hline \multirow{2}{*}{ deli. vo. } & \multirow{2}{*}{ Jepth: } & \multirow{2}{*}{ Perforations } & \multirow{2}{*}{ r* } & \multirow{2}{*}{ cos } & \multirow{2}{*}{ Ana:ys:s } & \multicolumn{3}{|c|}{ Whter-ievel measurements } \\
\hline & & & & & & Dradoum & Recovery & Histaricel \\
\hline $2 / 7-3 \mathrm{AI}$ & 560 & $200-560$ & - & Dr1llers & yes & yes & yes & yes \\
\hline $2 / 7-2 \mathrm{Cl}$ & 400 & $2 /$ & - & Drillers & yes & yes & yes & yes \\
\hline & & & & & & & & \\
\hline & & & & & & & & \\
\hline & & & & & & & & \\
\hline
\end{tabular}

other Dat: and Reanrks 2. Perforations, 149-152, 189-192, 239-259, 261-268,

271-305, 325-330, 356-377 ft. Prepump1ng water level in 3AI, 25.45 it

below measuring point.

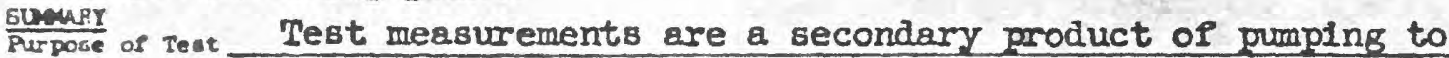
develop well.

Mquiser Thickres

speciese capactey $35.8^{3}$ Aquafor suturated Thackess

Mehad or Armiges: ITorequilibrium

cuerririent or Estlmated.

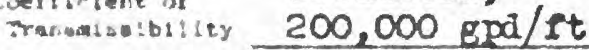
Inmentsi:

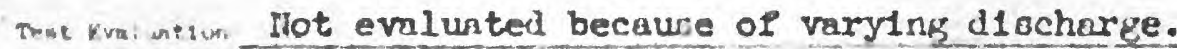

3. Discharge 1818 gra at 108 min. after pumping etnrted. 
U.S. FEOLCE:CA: SIRVEY

GRCUNC WATER BRLNCH

AQUTHER-TEST CCHPILATICN

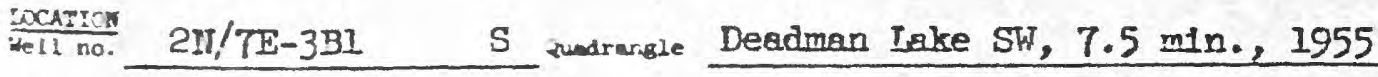

Location About 15 miles northwest of Twentynine Palms, San Bernardino County

Grownd water Basin Colorado River Basin, 7-

Geologte Fornetion Alluvium

Dare of Test Jen. 12, 1953 If Agency Conducting test USCS, F. S. Riley

Source of Teat Dats USGS (GW), Sacramento

ZUPPIC EATA

Pun Type, Power source and Bation

Use

D1 scharge

1)

Maximian Draidown

Pt. after

min.

cther tat 1. Pumped for $2400 \mathrm{~min}$. at discharge rates varying from about $900 \mathrm{gpm}$ to about $1500 \mathrm{gpm}$.

VELL DATA

\begin{tabular}{|c|c|c|c|c|c|c|c|c|}
\hline \multirow{2}{*}{ deli no. } & \multirow{2}{*}{ Depth } & \multirow{2}{*}{ Perforat:ons } & \multirow{2}{*}{$r *$} & \multirow{2}{*}{$\therefore$} & \multirow{2}{*}{ Ans:ysis } & \multicolumn{3}{|c|}{ Water-leve! measurements } \\
\hline & & & & & & Eraudom & Recovery & Historlea? \\
\hline $2 / 7-3 B I$ & 700 & $260-690$ & -- & Drillers & yes & yes & yes & yes \\
\hline - & & & & & & 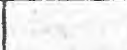 & & \\
\hline & & & & & & & & \\
\hline & & & & & & & & \\
\hline
\end{tabular}

cther Dats and Remarks - Prepumpling water level in 3Bl, $105,66 \mathrm{ft}$ below measuring point.

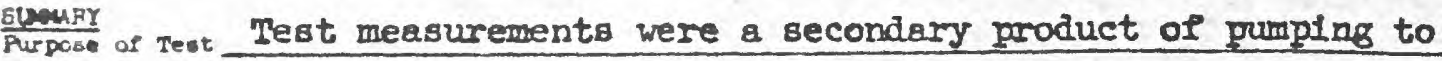
develop well

Aquifer Thicknes Aqus ser Saturatent oncksess

Specirte Capaity CSGS Fomation Yiels Fuceor

Metroct of Araigad: Nonequilibrium

Cavericlent of $25,000 \mathrm{gpd} / \mathrm{ft}$ (t)

The rn wion lot evalunted because of varled discharge. 
U.S. IECLUITHA SUEVEY

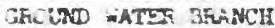

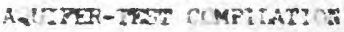

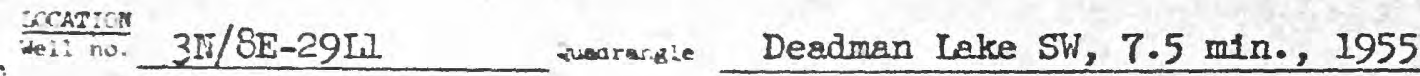

Locion About $14 \frac{1}{2}$ miles northwest of Twentynine Palms, San Bernardino County

Ground water Basin Colorado River Basin, 7-

Geologie Fornation Alluvium

Date of Test Dec. 8, 1952 I/ Amerny Conductir.s Test USGS, F. S. RIley

Source of Test Deta USGS (GW), Sacramento

Pisc:

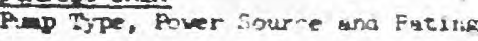

loe Public Supplycischarge I] Maxinize Drastom ft. neter nin.

other dat 1. Pumped for about $1200 \mathrm{~min}$. at discharge rates varylng from $100 \mathrm{gman}$ to $2,000 \mathrm{ggm}$.

WELI IAEA

\begin{tabular}{|c|c|c|c|c|c|c|c|c|}
\hline \multirow{2}{*}{$-1 . s$} & \multirow{2}{*}{ Septr: } & \multirow{2}{*}{ Rerforations } & \multirow{2}{*}{$\mathrm{r}^{*}$} & \multirow{2}{*}{ we } & \multirow{2}{*}{ Arsa:ysio $y$} & \multicolumn{3}{|c|}{ Whicr-ievel measurementa } \\
\hline & & & & & & Eravedom & Secusery & Historical \\
\hline $3 / 8-29[1$ & 600 & $270-590$ & -- & Drillers & yes & yes & yes & yes \\
\hline $3 / 8-29 \mathrm{Cl}$ & 800 & 2) & 2397 & Drillers & yes & yes & no & yes \\
\hline . & & & & & & & & \\
\hline & & & & & & & & \\
\hline & & & & & & & & \\
\hline
\end{tabular}

ther Data and Rexurks 2. Perforated 500-523, 540-565, 584-605, 640-646,

660-684 ft. Prepumping water level in 29II, 103.39 ft below measuring point.

Furpose of Teet Test measurements were a secondary product of pumping to

develop well.

Aquifer Intckrese

Average

specurse capwets 35.7

Aqusent Coturnted miekres?

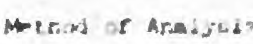

nonequilibrium

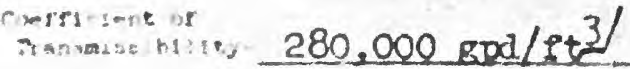

... Not evmluated because of varled discharge.

3. Eat1nuted on atep drawlogent. 
[Duplicate data from Dec. 8 form except as noted]
T.5. GEOTEGICAL SURVEY

CROUNO WATER BRANCH

AVIVET-IEST COMPILATION

WII: no. 3N/8E-29LI S Rumirangie

Locetion

Cround weter Basin

Geologte Formation

Date of Test Aug. 6, 1953735 mingrency Conductirg Test USGS, F. S. R1ley

Source of Test Data

PUXPIYC DATA

Pung Type, Pover Source and Fating

Average

Use

Discharge $890 \mathrm{gpm}$

Maximue Drawtom

Pt. after $\min$.

Other deta

\begin{tabular}{|c|c|c|c|c|c|c|c|c|}
\hline \multirow{2}{*}{ Heil so. } & \multirow{2}{*}{ Depth } & \multirow{2}{*}{ Perforations } & \multirow{2}{*}{$r$} & \multirow{2}{*}{$\mathrm{LOB}_{\mathrm{B}}$} & \multirow{2}{*}{ Anaiysis } & \multicolumn{3}{|c|}{ water-level messurements } \\
\hline & & & & & & Iravdoum & Recovery & Historical \\
\hline $3 / 8-29$ II & -- & -- & - & - & -- & No & Yes & No \\
\hline & & & & & & 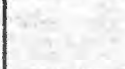 & & \\
\hline & & .3 & & 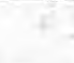 & & & & \\
\hline & & & & & & & & \\
\hline & & & & & & & & \\
\hline
\end{tabular}

$r^{*}$ - distafice fram pumped wil to observition weil in feet.

Other Lata and Remarks

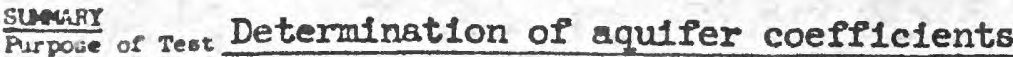

Aqusfer Thickness Aquifer saturated mickness

3peifle Capactey KGG: Formation Yield Factor

Methoul or Anniy $y=10$ Nonequi11brium

Coerrtelent of $270,000 \mathrm{gpd} / \mathrm{ft}$ ericlent or corace

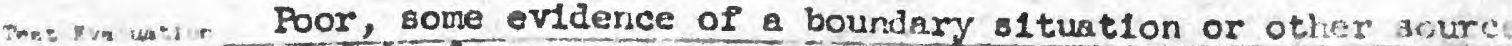
of Interference. 
Spec1fic Capacity Tests in the Threntynine Palms area

\begin{tabular}{|c|c|c|c|c|c|}
\hline Vell numioer & $\begin{array}{l}: \\
: \text { ieli depth } \\
: \quad \text { (ieet) } \\
:\end{array}$ & $\begin{array}{l}\text { :Date teste } \\
: \\
:\end{array}$ & $\begin{array}{l}\text { : } \\
: \text { Discherge } \\
: \quad(\text { gpn }) \\
:\end{array}$ & $\begin{array}{l}: \\
\text { e:Drawdorn } \\
:(\text { feet }) \\
:\end{array}$ & $\begin{array}{l}: \quad \text { Speciflc } \\
: \quad \text { capecity } \\
:(\mathrm{gam} / \mathrm{ft} \text { of } \mathrm{da})\end{array}$ \\
\hline $1 / 9-4 i 1$ & 136 & $4-7-52$ & 256 & & 6.2 \\
\hline $5 \mathrm{GI}$ & 500 & $0-4-61$ & 780 & 44 & 17.8 \\
\hline $2 / 7-2 \mathrm{CI}$ & 400 & & 346 & & 42.7 \\
\hline $3 A I$ & 560 & $5-23-61$ & 920 & 14 & 67.7 \\
\hline 331 & 700 & $5-23-61$ & 910 & 37 & 24.7 \\
\hline 4111 & 500 & & 267 & & 12.2 \\
\hline $14 \pi[$ & 644 & & 76 & & 1.4 \\
\hline $2 / 8-24 \mathrm{HI}$ & 320 & & 190 & & 1.9 \\
\hline $3 / 7-13 i 1$ & 188.5 & & $2 \varepsilon 2$ & & 11.2 \\
\hline $1 E D 1$ & 384.5 & & 263 & & 13.7 \\
\hline 3181 & 430 & & 262 & & 30.5 \\
\hline $35 \mathrm{P2}$ & & $5-23-61$ & 1,700 & 20 & 82.9 \\
\hline $3 / 8-174$ & 512 & & 216 & & 1.6 \\
\hline $29 \mathrm{Cl}$ & 800 & & 250 & & 8.6 \\
\hline 29LI & 600 & $6-6-61$ & 1,060 & 12 & 86.8 \\
\hline 3331 & 526 & & 350 & & 21.6 \\
\hline 3401 & 400 & & 256 & & 13.5 \\
\hline
\end{tabular}




\section{REFEREIGE LIST}

A. McClelland, E. J., 1962, Aquifer-test complletion for the San Joaquin Valley, Callf.: U.S. Geologlcal Survey, Sacramento, Cal1f., open-f1le dupl. rept., 38 p., 2 f1gs.

B. 1963, Aquifer-test compllation for the Hojave Desert region, Cal1f.: U.S. Geological Survey, Sacramento, Calif., open-file dupl. rept., 29 p., 3 flgs.

c. 1963, Aquifer-test compllation for the upper Sante Ana

Valley area, San Bernardino County, Callf.: U.S. Ceologtcal Survey, Sacramento, Cal1f., open-f1le dupl. rept., 29 p., 2 flgs.

1963, Aquifer-test compllation for northern Callfornte: J.S. Geolog1cal Survey, Sacramento, Callf., open-file dupl. rept., 24 p., 4 flgs.

E. 1963. Aquifer-test complation for the Central Coastal

Región, Callfornia: U.S. Ceological Survey, Sacramento, Cal1f., open-f1le dupl. rept., , 53 p., 2 flgs.

T. 1963. Aquifer-test compliation for San Diego Reglon, Callfornia: U.S. Geologfcal Survey, Sacramenta, Callf., open-f1le dupl. rept., 19 p., 2 18g. 


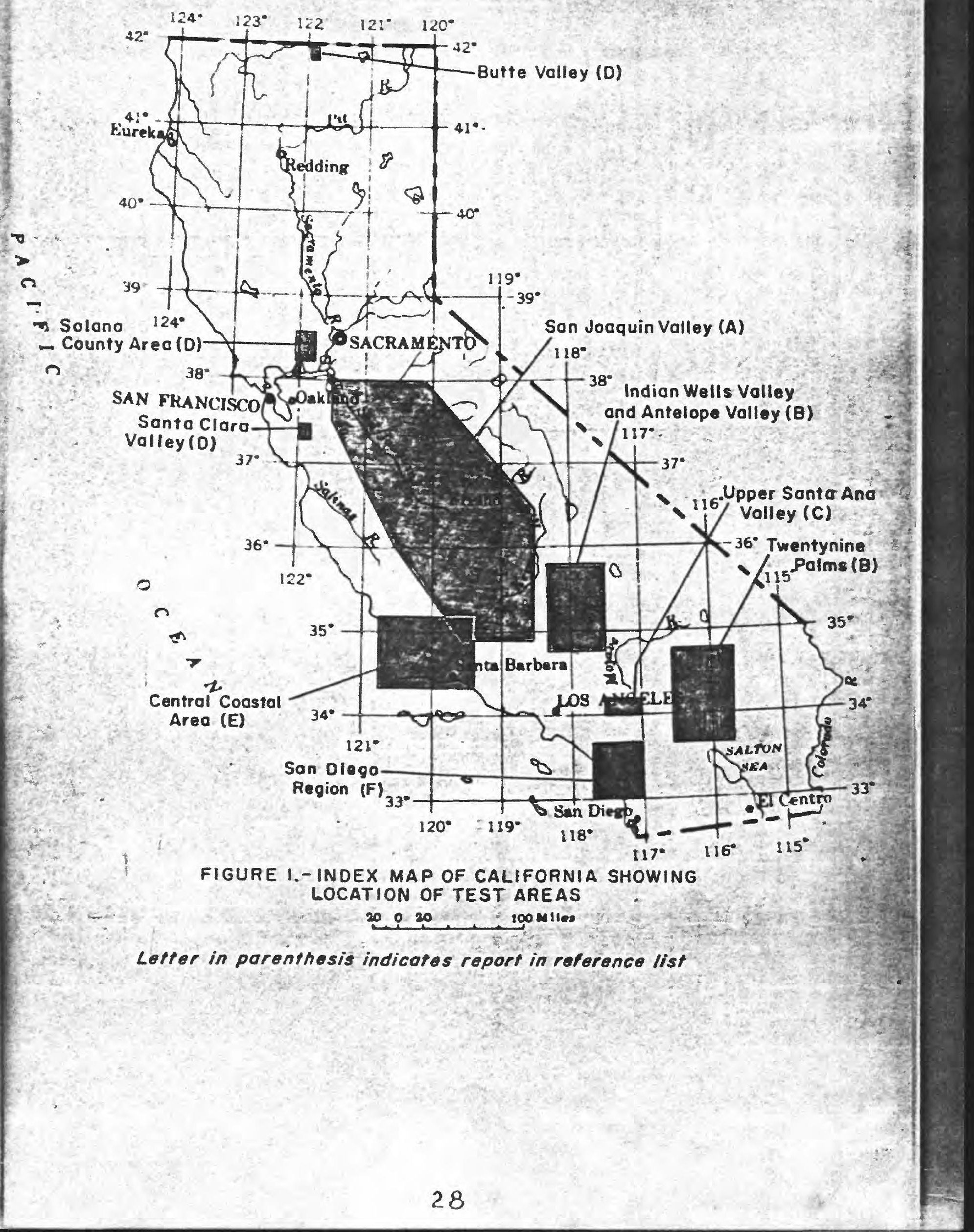




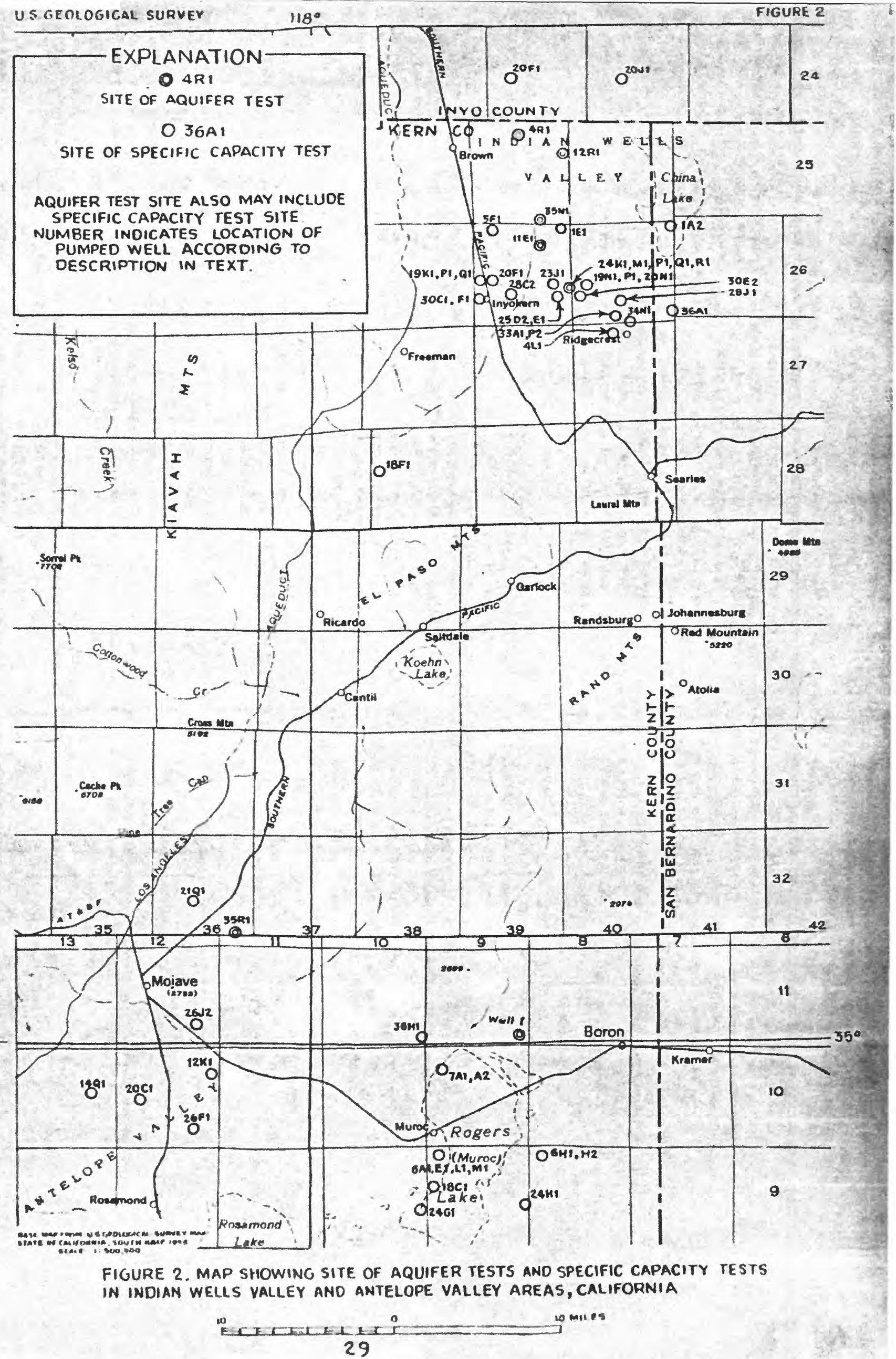


U.S. GEOLOGICAL SURVEY

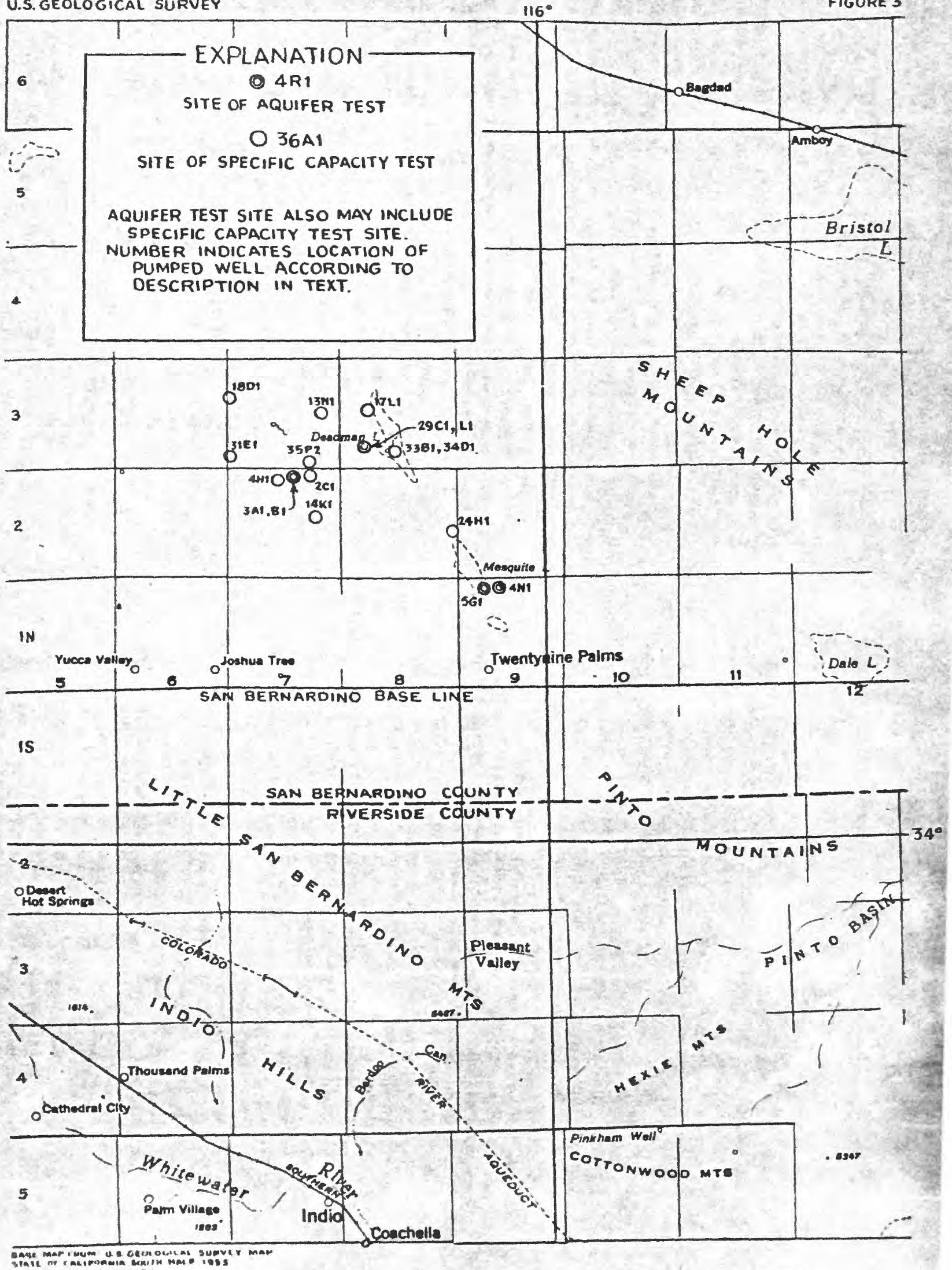

FIGURE 3. MAP SHOWINO SITE OF AQUIFER TESTS AND SPECIFIC CAPACITY TESTS IN THE TWENTYNINE PALMS AREA, CALIFORNIA 